\title{
Sensitivity-Enhanced Temperature Sensor Based on PDMS-Coated Long Period Fiber Grating
}

\author{
Qi Wang ${ }^{1,2, *}$, Chao Du ${ }^{1}$, Jiaming Zhang ${ }^{1}$, Riqing Lv ${ }^{1}$, Yong Zhao ${ }^{1,2}$ \\ ${ }^{1}$ College of Information Science and Engineering, Northeastern University, Shenyang 110819, China \\ ${ }^{2}$ State Key Laboratory of Synthetical Automation for Process Industries (Northeastern University), Shenyang 110819 , \\ China \\ *Corresponding author Email: wangqi@ise.neu.edu.cn
}

Corresponding Author: Qi Wang

Qi Wang is with College of Information Science and Engineering, Northeastern University, and State Key Laboratory of Synthetical Automation for Process Industries (Northeastern University).

Email:wangqi@ise.neu.edu.cn

Telephone number: $+86-13889195280$

Mailing address:

Northeastern University, College of Information Science and Engineering

P. O. Box 321, Shenyang, Liaoning, 110819, China

Chao Du, Jiaming Zhang and Riqing Lv are with College of Information Science and Engineering, Northeastern University.

Mailing address:

Northeastern University, College of Information Science and Engineering

P. O. Box 321, Shenyang, Liaoning, 110819, China

Yong Zhao is with College of Information Science and Engineering, Northeastern University, and State Key Laboratory of Synthetical Automation for Process Industries (Northeastern University).

\section{Mailing address:}

Northeastern University, College of Information Science and Engineering

P. O. Box 321, Shenyang, Liaoning, 110819, China 


\title{
Sensitivity-Enhanced Temperature Sensor Based on PDMS-Coated Long Period Fiber Grating
}

\author{
Qi Wang ${ }^{1,2, *}$, Chao Du ${ }^{1}$, Jiaming Zhang ${ }^{1}$, Riqing $\mathrm{Lv}^{1}$, Yong Zhao ${ }^{1,2}$ \\ ${ }^{1}$ College of Information Science and Engineering, Northeastern University, Shenyang 110819, China
}

${ }^{2}$ State Key Laboratory of Synthetical Automation for Process Industries (Northeastern University), Shenyang 110819, China

*Corresponding author Email: wangqi@ise.neu.edu.cn

\begin{abstract}
A sensitivity-enhanced temperature sensor based on a poly-dimethylsiloxane (PDMS)-coated long period fiber grating (LPFG) has been proposed and experimentally investigated. By embedding the LPFG in a temperature-sensitive elastomeric polymer, the temperature sensitivity of the proposed sensor could be effectively improved by 4 times higher than those of the conventional bare LPFG sensors due to the high thermo-optic coefficient (TOC) of PDMS. It can be found that the temperature sensitivities of higher-order modes are higher than those of lower-order modes by analyzing transmission spectra characteristics of the sensor. Because of LPFG is sensitive to surrounding refractive index (RI), the PDMS-coated LPFG will have a high temperature sensitivities of $255.4 \mathrm{pm} /{ }^{\circ} \mathrm{C}$ in the range of $20^{\circ} \mathrm{C}$ to $80^{\circ} \mathrm{C}$. Due to the high measurement resolution of $0.078^{\circ} \mathrm{C}$, the sensor is promising to be applied to the fields that high-precision temperature measurement is required.
\end{abstract}

Keywords: long period fiber grating, optical fiber sensors, temperature measurement, sensitivity-enhanced.

\section{Introduction}

Temperature is closely related to the physical and chemical characteristics of material. The measurement of temperature has great significance to the application field of physical, biological and chemical. For decades, fiber sensors have been widely studied due to their small volume, fast response, high sensitivity, resistance to electromagnetic interference and the advantage of long distance detection ${ }^{[1]}$. Since characteristics of material has a strong dependence on temperature, it is essential to measure temperature in practical applications ${ }^{[2]}$. At present, various of temperature sensors based on optical fiber structure have been proposed and demonstrated, such as Sagnac ${ }^{[3,4]}$, Fabry-Perot ${ }^{[5-8]}$, fiber Mach-Zehnder interferometers ${ }^{[9-10]}$, fluid-filled photonic crystal fiber ${ }^{[11-12]}$, etc.

In addition, due to the advantages of compact structure, simple fabrication, and easy construction of long range monitoring system, fiber Bragg grating (FBG) sensors are also used for the measurement of 
temperature $^{[13]}$. But the temperature sensitivity of FBG is low due to the low thermal expansion coefficient (TEC) of silica with temperature variation. In order to enhance the temperature sensitivity of FBG sensors, Park et al. proposed a PDMS-coated optical fiber Bragg grating sensor ${ }^{[14]}$. The temperature sensitivity of the proposed structure could be effectively improved by 4.2 times higher than those of the conventional bare-type FBG sensors. Although the temperature sensitivity of LPFG sensor is much higher than that of FBG, it hardly meet the requirement of high-precision measurement due to a low thermo-optic coefficient (TOC) of silica ${ }^{[15]}$.

With the development of material, there have been many reports about the measurements of temperature by using materials with a high TOC $^{[16-18]}$. The disadvantage of these sensitive temperature sensors is that they employ liquids or alcohols which may leak or evaporate ${ }^{[19]}$, and it's difficult to be fabricated due to complicated sensing structure. The materials with a high TOC will produce a large RI change with surrounding temperature variation, so it can achieve the measurement of temperature by monitoring RI change. Compared with FBG, LPFG can couple the core mode to the forward propagating cladding modes, and shows relatively high RI sensitivity because of the strong interaction between cladding modes and surrounding medium, so it can be used for the measurement of temperature.

The PDMS can be formed into any shape with various molds due to the low elastic modulus and high Poisson's ratio, which is easy to be integrated with optical fiber sensors. Compared with other TOC materials, it also has advantages of non-toxic, better transmittance, lower cost and relatively high chemical inertness. In this letter, we proposed and fabricated a sensitivity-enhanced temperature sensor based on a PDMS-coated LPFG. The experiments showed that the temperature sensitivity of the proposed sensor could be effectively improved by embedding the LPFG in a temperature-sensitive elastomeric polymer PDMS (with a high TOC of $-4.5 \times 10^{-4} /{ }^{\circ} \mathrm{C}$ ). The temperature sensitivity of the proposed sensor can reach $255.4 \mathrm{pm} /{ }^{\circ} \mathrm{C}$ with a LPFG length of $2.4 \mathrm{~cm}$ and a grating period of $480 \mu \mathrm{m}$, which is 4 times higher than that of the conventional bare LPFG sensors, in the range of $20^{\circ} \mathrm{C}$ to $80^{\circ} \mathrm{C}$. It has a high measurement resolution of $0.078^{\circ} \mathrm{C}$ and advantages of easy fabrication, compact structure, wide measurement range, good linearity and stability.

\section{Sensing Structure and Measurement Principle}

The schematic diagrams of the PDMS-coated LPFG sensing experimental setup are shown in Fig. 1. A broadband light source (ASE) with a wavelength range from 1300 to $1700 \mathrm{~nm}$ and an optical spectrum analyzer (OSA) with a wavelength resolution of $20 \mathrm{pm}$ were employed to monitor the transmission spectra of our proposed sensor. The sensing head was placed in a temperature-controlled chamber and fixed with two fiber holders to keep it straight for preventing the interference from external tiny stress. 


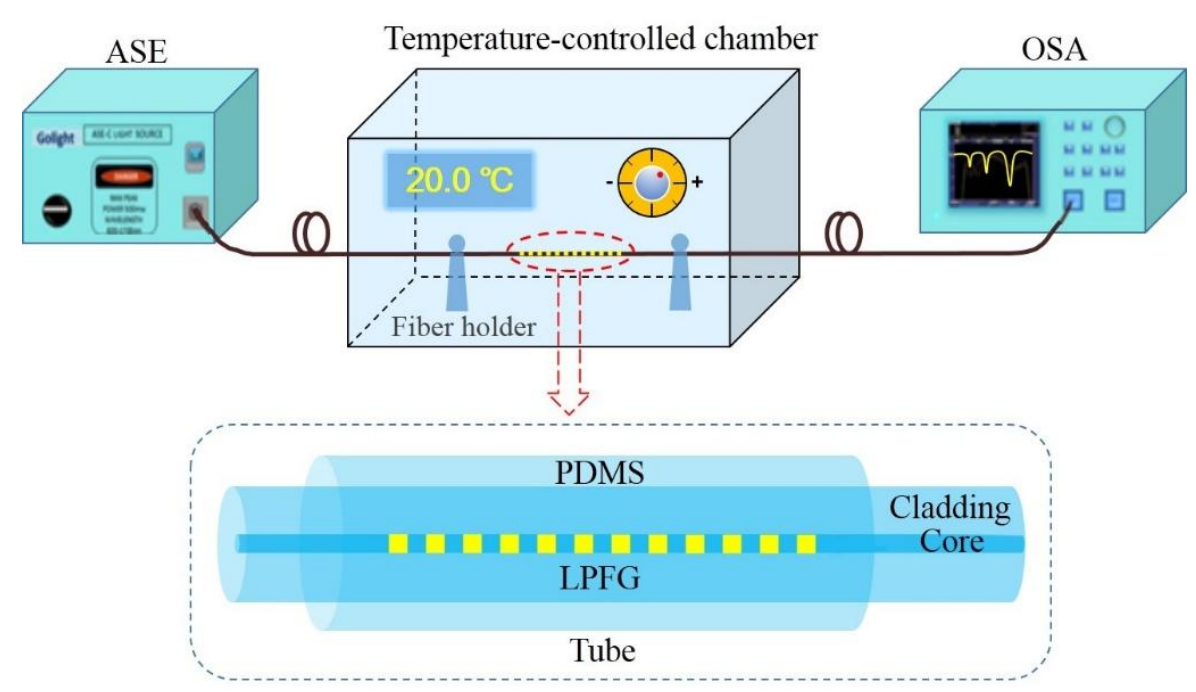

Fig. 1. The schematic diagrams of the PDMS-coated LPFG sensing experimental setup.

The inset is the enlarged structure of temperature sensor.

In our proposed sensor, the LPFG was inscribed in the common single-mode fiber (SMF, Corning SMF-28) with a grating period of $480 \mu \mathrm{m}$ through UV exposure. Then the LPFG was encapsulated in a capillary tube, and the PDMS solution with a 10:1 mixing ratio of precursor of elastic material and hardener was filled into the tube by capillary force ${ }^{[20]}$. Both ends of the tube were sealed with glue. The length of the polymer was more than $30 \mathrm{~mm}$, which ensured that the LPFG was completely immersed in the PDMS. Later, the pre-polymer of the mixture was polymerized by baking it in the thermostat for several hours ${ }^{[21]}$. Finally, the PDMS-coated LPFG temperature sensor was obtained.

The LPFG can couple the core mode to the forward propagating cladding modes. The coupling between the core mode and the $m$ th cladding mode matches the phase-matching conditions:

$$
\beta_{c o}-\beta_{c l, m}=2 \pi / \Lambda
$$

where $\beta_{c o}$ and $\beta_{c 1, m}$ are the propagation constants of the core mode and the $m$ th order cladding mode, respectively. $\Lambda$ is the grating period. Therefore, resonant wavelength $\lambda_{\text {res }}$ is determined by:

$$
\lambda_{\text {res }}=\left(n_{e f f}^{c o}-n_{e f f}^{c l, m}\right) \Lambda
$$

where $n_{\text {eff }}^{c o}$ and $n_{\text {eff }}^{c l, m}$ are the effective refractive indices of core mode and the $m$ th cladding mode, respectively.

We fabricated a LPFG with a length of $2.4 \mathrm{~cm}$ and a grating period of $480 \mu \mathrm{m}$, and then connected it to ASE and OSA by using two commercial SMF adapters. The transmission spectrum of bare LPFG in air at room temperature is shown in Fig. 2. The four resonant dips of transmission spectrum were induced by the coupling of core mode $\mathrm{LP}_{01}$ and cladding modes $\mathrm{LP}_{02}$ (with a resonant wavelength of $1358.29 \mathrm{~nm}$ ), $\mathrm{LP}_{03}$ (with a resonant wavelength of $1392.46 \mathrm{~nm}$ ), $\mathrm{LP}_{04}$ (with a resonant wavelength of $1456.78 \mathrm{~nm}$ ), $\mathrm{LP}_{05}$ (with a 
resonant wavelength of $1579.39 \mathrm{~nm})$, respectively.

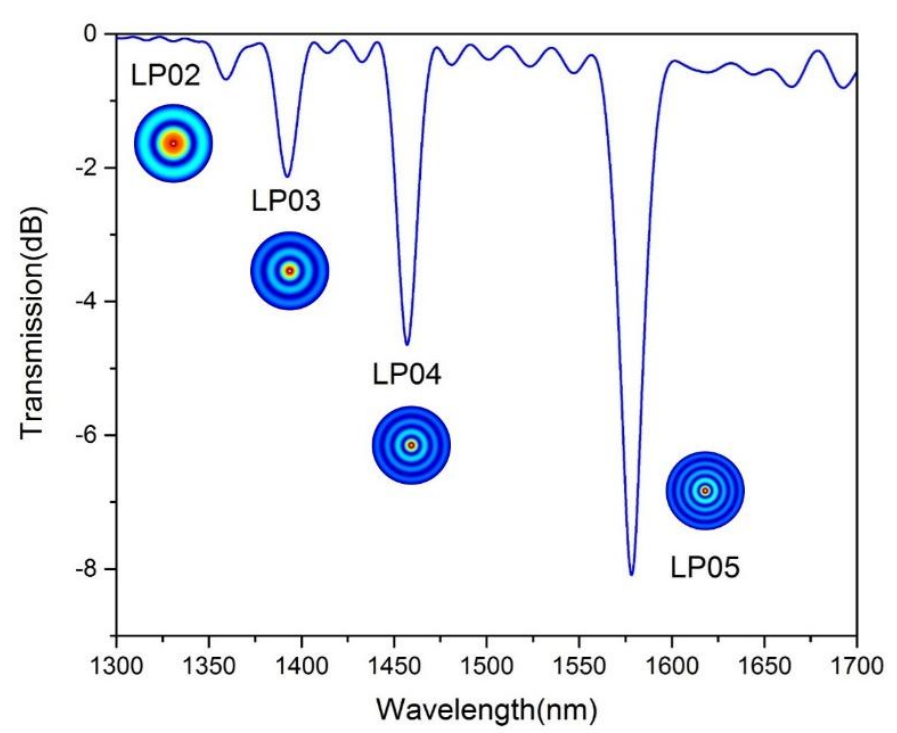

Fig. 2. The transmission spectrum of bare LPFG in air at room temperature.

Actually, the temperature sensitivities of conventional bare LPFG sensors are mainly determined by the thermal expansion and thermo-optic effects of silica. Considering temperature, the phase-matching conditions is given by:

$$
\beta_{c o}(\lambda, T)-\beta_{c l, m}(\lambda, T)=2 \pi / \Lambda
$$

where $\beta_{c o}(\lambda, T)$ and $\beta_{c l, m}(\lambda, T)$ are the propagation constants of core mode and the $m$ th order cladding mode when temperature is $T$ and resonant wavelength is $\lambda . \Lambda$ is the grating period when the temperature is $T$, as shown in Eq. (4).

$$
\Lambda=(1+\alpha \Delta T) \Lambda_{0}
$$

Where $\alpha$ represents the TEC. The temperature sensitivity $K_{T}$ is given by:

$$
K_{T}=\frac{d \lambda_{r e s}}{d T}=\left(\frac{\partial n_{e f f}^{c o}}{\partial T}-\frac{\partial n_{e f f}^{c l, m}}{\partial T}\right) \Lambda+\left(n_{e f f}^{c o}-n_{e f f}^{c l, m}\right) \frac{\partial \Lambda}{\partial T}=\lambda_{r e s} \frac{\alpha+\frac{\zeta_{e f f}^{c o} n_{e f f}^{c o}-\zeta_{e f f}^{c l, m} n_{e f f}^{c l, m}}{n_{e f f}^{c o}-n_{e f f}^{c l, m}}}{1-\frac{\lambda_{r e s}}{n_{e f f}^{c o}-n_{e f f}^{c l, m}} \frac{\partial\left(n_{e f f}^{c o}-n_{e f f}^{c l, m}\right)}{\partial \lambda}}=\gamma \lambda_{r e s}\left(\alpha+\frac{\zeta_{e f f}^{c o} n_{e f f}^{c o}-\zeta_{e f f}^{c l, m} n_{e f f}^{c l, m}}{n_{e f f}^{c o}-n_{e f f}^{c l, m}}\right)
$$

in which the first term means the influence of the TOC and the second term means the influence of the TEC, $\gamma$ represents the waveguide dispersion factor, $\zeta_{\text {eff }}^{c o}$ and $\zeta_{\text {eff }}^{c l, m}$ are the effective refractive TOCs of core mode and the $m$ th cladding mode, respectively ${ }^{[15]}$. The temperature sensitivity of bare LPFG can hardly meet the requirement of high-precision measurement due to low TOC $\left(8.6 \times 10^{-6} /{ }^{\circ} \mathrm{C}\right)$ and $\mathrm{TEC}\left(5.5 \times 10^{-7} /{ }^{\circ} \mathrm{C}\right)$ of silica. The thermal expansion effect changes the grating period and the thermo-optic effect changes the effective refractive indices of the core mode and cladding modes at the resonant wavelengths. Because of the TEC is an order of magnitude higher than TOC, the thermo-optic effect is the dominant factor of 
temperature sensitivity.

The effective refractive indices of cladding modes are usually affected by the RI of surrounding medium, but the effective refractive index of core mode changes little. The resonant wavelength $\lambda_{\text {res }}$ will shift, when the $m$ th cladding mode effective refractive index $n_{e f f}^{c l, m}$ changes. Therefore, the RI of surrounding medium $n$ can be measured by monitoring the shift of resonance wavelength $\lambda_{\text {res }}$. The RI measurement sensitivity $K_{n}$ is given by:

$$
K_{n}=\frac{d \lambda_{\text {res }}}{d n}=\left(\frac{\partial n_{e f f}^{c o}}{\partial n}-\frac{\partial n_{e f f}^{c l, m}}{\partial n}\right) \Lambda
$$

Therefore, if the bare LPFG is surrounded by a material with a high TOC, as for example, PDMS, a small temperature change can induce a prominent shift of the resonance dips. Therefore, we can propose and fabricate a sensitivity-enhanced temperature sensor based on a PDMS-coated LPFG. The mechanism of the PDMS-coated LPFG is to transfer the temperature response into the refractive index response of the surrounding PDMS due to the high TOC.

\section{Experimental Results and Discussions}

Two LPFGs with the same parameters were inscribed in our experiments, one is bare and the other is coated with PDMS. Light from the ASE was coupled to the LPFG and the transmission spectra were recorded by OSA. Fig. 3 shows the transmission spectra of the device when the LPFG was surrounded by air and PDMS at room temperature $\left(20^{\circ} \mathrm{C}\right)$. The RI of PDMS is about 1.42 which is higher than that of air (RI=1), so transmission spectra of the two LPFGs are different due to the phase matching conditions of the interference are changed by surrounding RI.

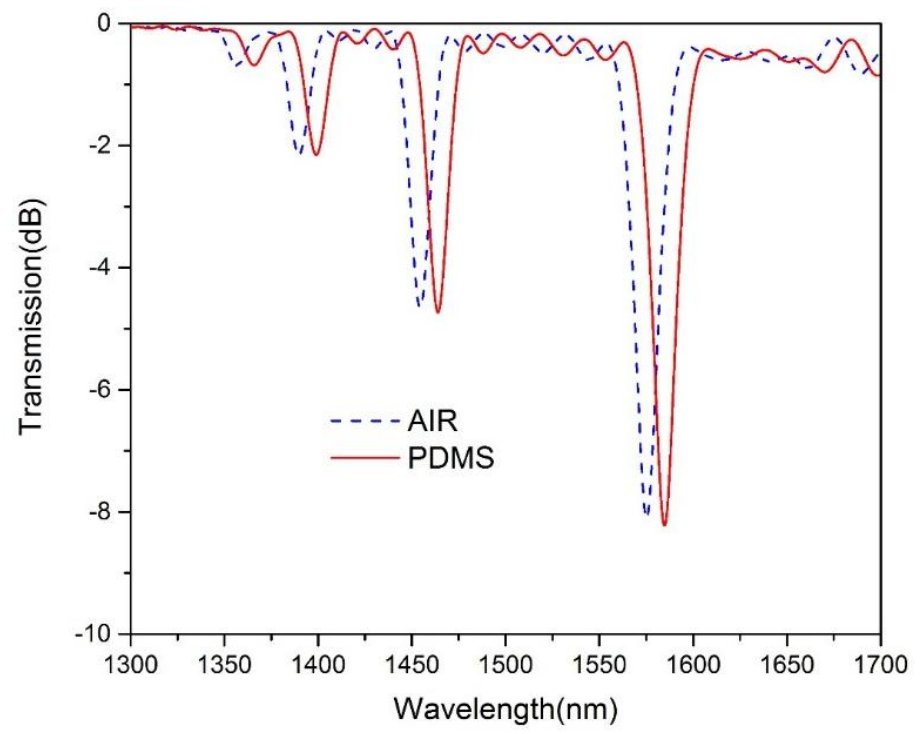

Fig. 3. The transmission spectra of the device when the LPFG was surrounded by air and PDMS at room temperature $\left(20^{\circ} \mathrm{C}\right)$. 
The temperature sensing characteristics of the bare LPFG should be investigated, firstly. The bare LPFG sensor was placed into a temperature-controlled chamber which has a temperature resolution of $0.1{ }^{\circ} \mathrm{C}$. And then heated up from $20^{\circ} \mathrm{C}$ to $80{ }^{\circ} \mathrm{C}$ in steps of $10^{\circ} \mathrm{C}$. At each step, the temperature was kept for 20 minutes and the transmission spectrum of the sensor was recorded after it stabled. With temperature increase, the transmission spectrum moves to long wavelength, as shown in Fig. 4. The inset displays the shifts of wavelengths of Dip4.

The resonant wavelength shifts of four dips at different temperature are shown in Fig. 5. The wavelengths of resonant dips move toward longer wavelength direction due to the thermal expansion and thermo-optic effects of the silica. The fitting curves are also presented in Fig. 5, in which the temperature sensitivities for Dip1, Dip2, Dip3, and Dip4, are $39.5 \mathrm{pm} /{ }^{\circ} \mathrm{C}, 40.8 \mathrm{pm} /{ }^{\circ} \mathrm{C}, 44.8 \mathrm{pm} /{ }^{\circ} \mathrm{C}$, and $50.5 \mathrm{pm} /{ }^{\circ} \mathrm{C}$, respectively.

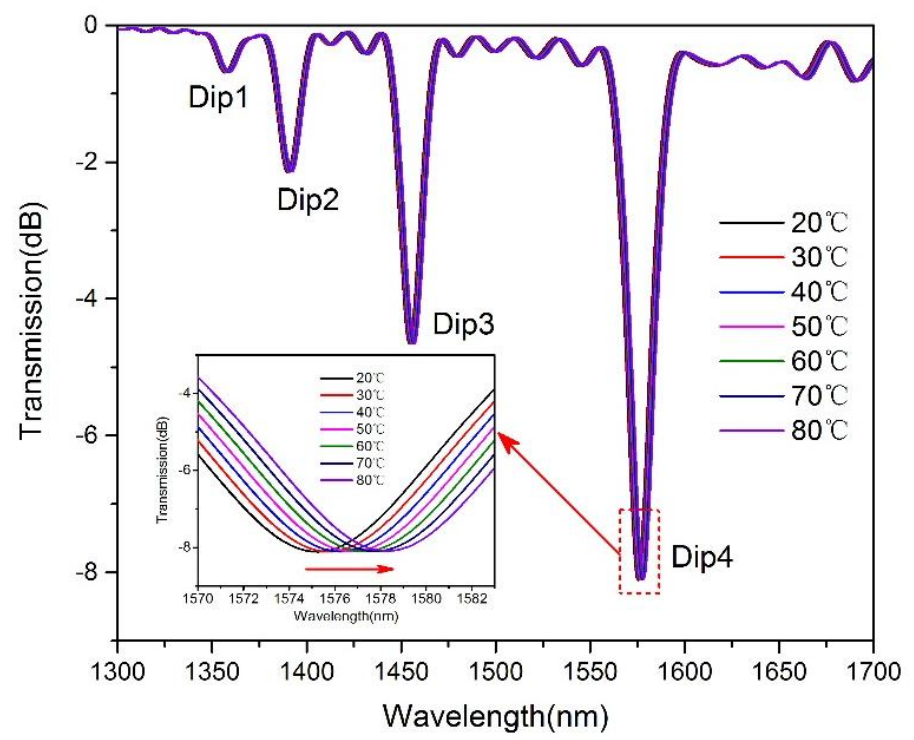

Fig. 4. Transmission spectrum of the sensor changes at different temperature.

Inset displays shifts of wavelength of Dip4.

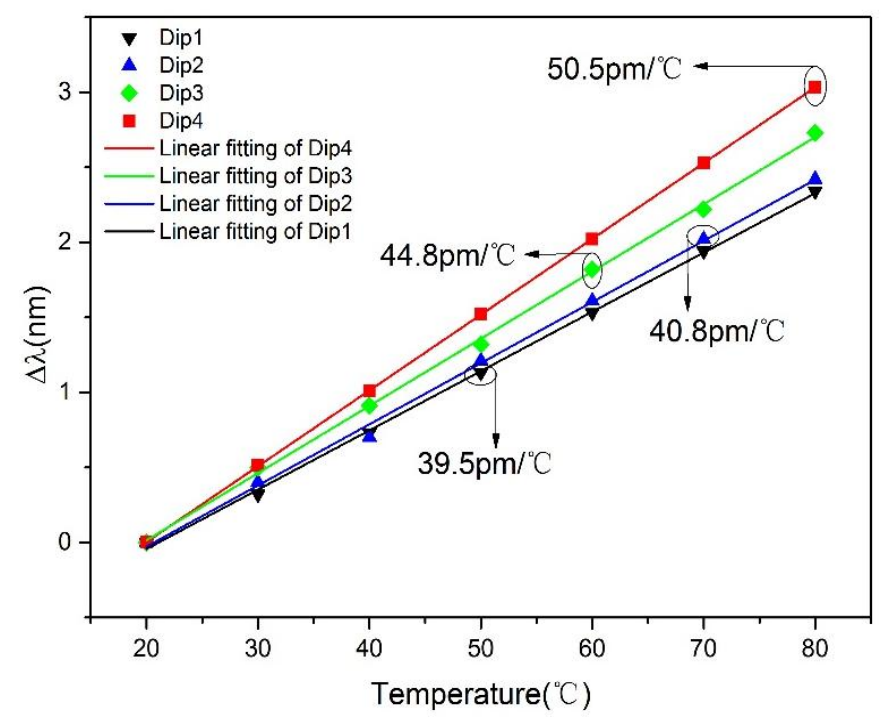

Fig. 5. Relationships between temperature and wavelength shift of the resonant dips 
Dip1, Dip2, Dip3 and Dip4, respectively.

In order to test the temperature sensitivity of our proposed device, the experiments above were repeated. With temperature increase, the transmission spectrum moves to long wavelength, as shown in Fig. 6. The inset displays the shifts of wavelengths of Dip4.

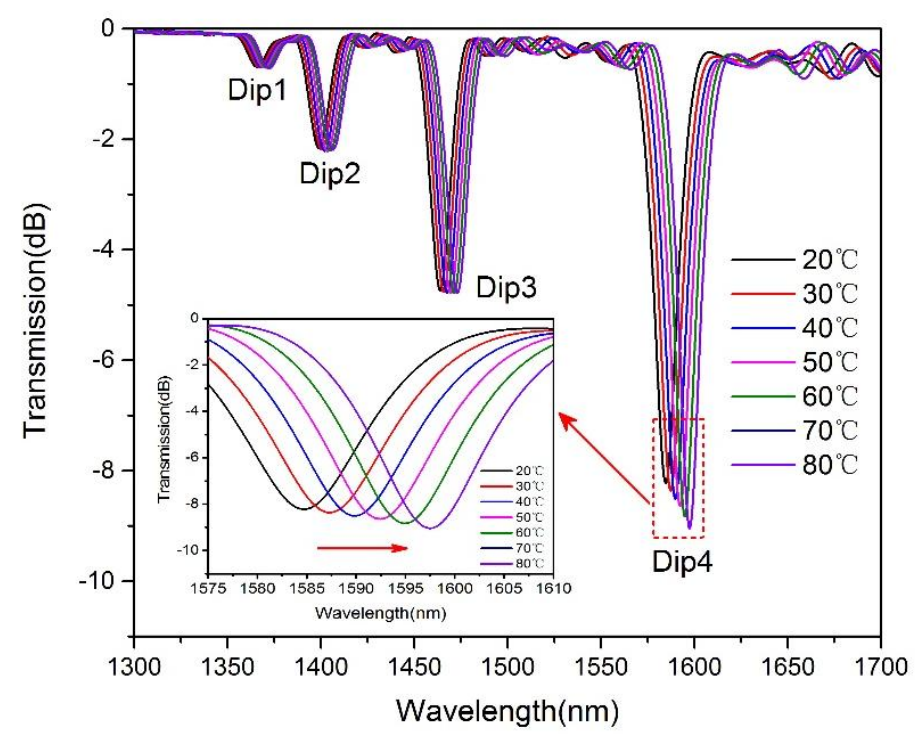

Fig. 6. Transmission spectrum of the sensor changes at different temperature.

Inset displays shifts of wavelengths of Dip4.

The resonant wavelength shifts of four dips at different temperature are shown in Fig. 7. The wavelengths of resonant dips move toward longer wavelength direction due to the PDMS exhibits a highly negative TOC $\left(-4.5 \times 10^{-4} /{ }^{\circ} \mathrm{C}\right)^{[22]}$, which is agree with the RI sensitivity characteristics of LPFG. The fitting curves are also presented in Fig. 7, in which the temperature sensitivities for Dip1, Dip2, Dip3, and Dip4, are $129.2 \mathrm{pm} /{ }^{\circ} \mathrm{C}, 147.3 \mathrm{pm} /{ }^{\circ} \mathrm{C}, 180.7 \mathrm{pm} /{ }^{\circ} \mathrm{C}$, and $255.4 \mathrm{pm} /{ }^{\circ} \mathrm{C}$, respectively. Compared with the conventional bare LPFG sensor, the temperature sensitivity of the proposed sensor could be effectively enhanced by coating PDMS.

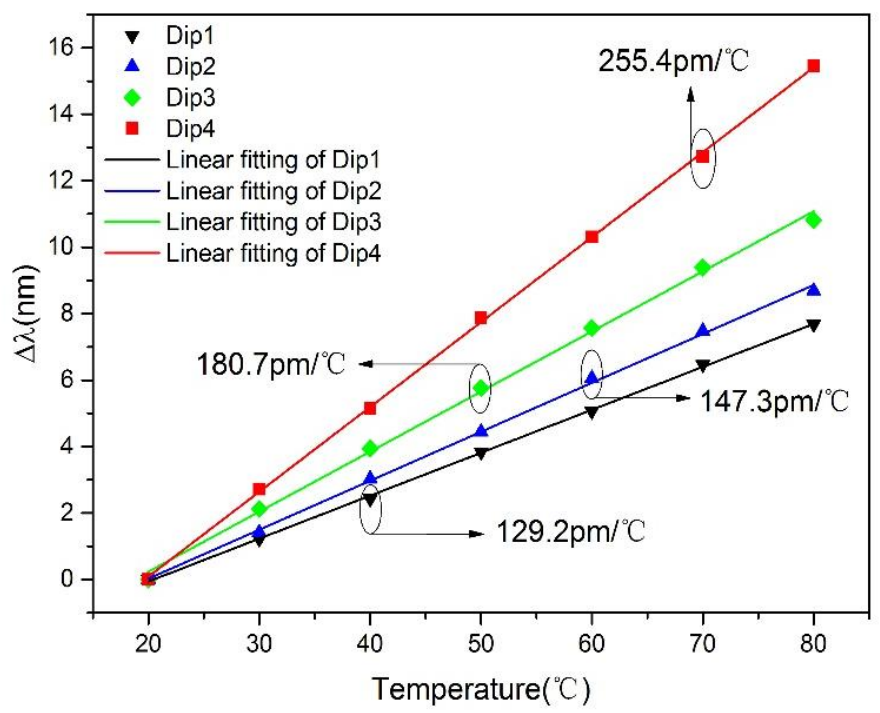

Fig. 7. Relationships between temperature and wavelength shift of the resonant dips 
Dip1, Dip2, Dip3 and Dip4, respectively.

After that, the temperature was decreased from $80{ }^{\circ} \mathrm{C}$ to $20{ }^{\circ} \mathrm{C}$ in steps of $10{ }^{\circ} \mathrm{C}$ by adjusting temperature-controlled chamber. At each step, the temperature was kept for 20 minutes and the transmission spectrum of the sensor was recorded after it stabled. With temperature decrease, the transmission spectrum moves to short wavelength, as shown in Fig. 8. The inset displays the shifts of wavelengths of Dip4.

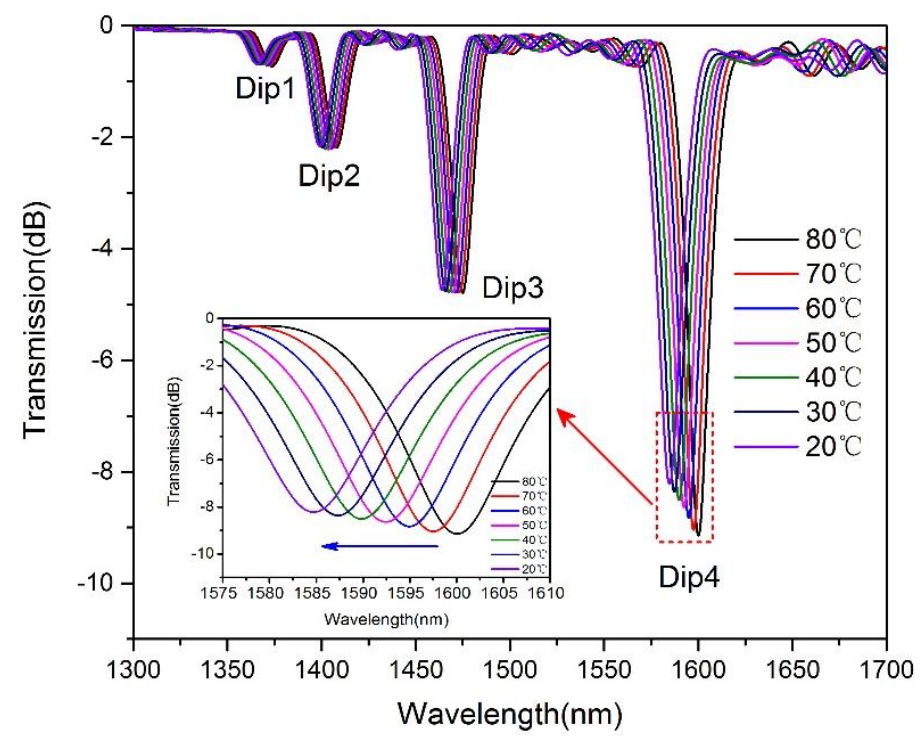

Fig. 8. Transmission spectrum of the sensor changes at different temperature.

Inset displays shifts of wavelengths of Dip4.

The resonant wavelength shifts of four dips at different temperature are shown in Fig. 9. The wavelengths of resonant dips move toward shorter wavelength direction, which is agree with the RI sensitivity characteristics of LPFG. The fitting curves are also presented in Fig. 9, in which the temperature sensitivities for Dip1, Dip2, Dip3, and Dip4, are $-129.3 \mathrm{pm} /{ }^{\circ} \mathrm{C},-147.5 \mathrm{pm} /{ }^{\circ} \mathrm{C},-181.2 \mathrm{pm} /{ }^{\circ} \mathrm{C}$, and -255.6 $\mathrm{pm} /{ }^{\circ} \mathrm{C}$, respectively. The temperature response results show that the temperature sensitivity is highly consistent with that of the heating, when the temperature decreases from $80{ }^{\circ} \mathrm{C}$ to $20{ }^{\circ} \mathrm{C}$. And the stability of our proposed device is good.

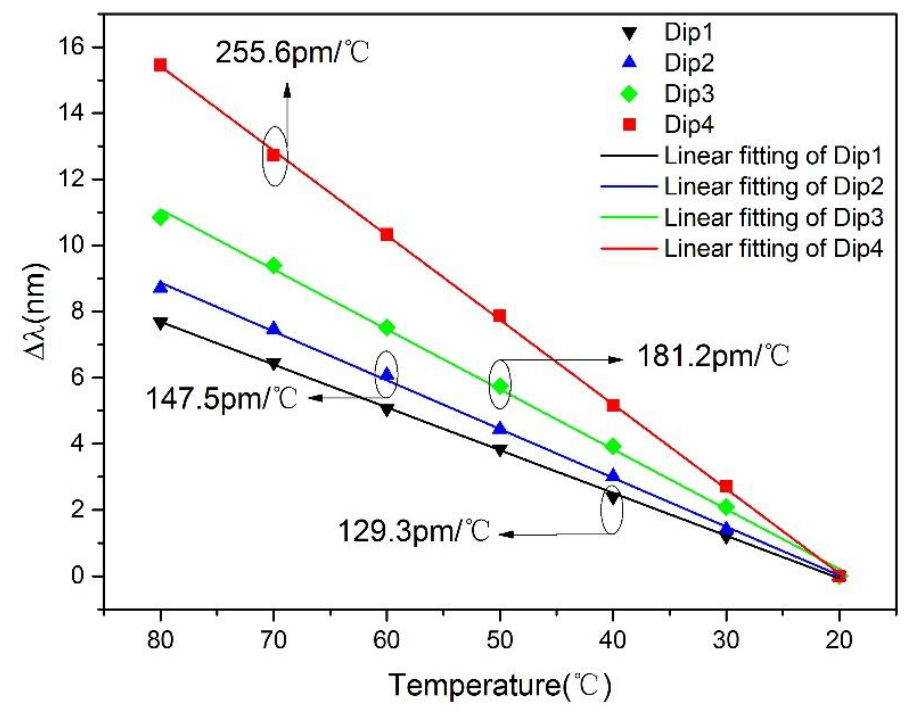


Fig. 9. Relationships between temperature and wavelength shift of the resonant dips

$$
\text { Dip1, Dip2, Dip3 and Dip4, respectively. }
$$

In summary, the temperature sensitivity of the PDMS-coated LPFG is higher than that of the bare LPFG. Within a temperature range of $20^{\circ} \mathrm{C}$ to $80^{\circ} \mathrm{C}$, the resonance wavelengths of the proposed sensor show linear temperature dependences. It can be found that the temperature sensitivities of higher-order cladding modes are higher than those of lower-order cladding modes because of the higher-order modes are much easier to be influenced by the surrounding environment.

\section{Conclusion}

We have proposed and experimentally demonstrated a high sensitivity temperature sensor based on a PDMS-coated LPFG. Experimental results show that a high temperature sensitivity of $255.4 \mathrm{pm} /{ }^{\circ} \mathrm{C}$ and a measurement resolution of $0.078^{\circ} \mathrm{C}$ are realized by embedding the LPFG in a temperature-sensitive elastomeric polymer PDMS, which have been effectively improved by 4 times higher than those of the conventional bare LPFG sensors. Due to the advantages of compact structure, wide measurement range, high sensitivity, good linearity and stability, the temperature sensor has great potential to be used in applications based on high precision temperature measurement, such as biological and chemical.

\section{Acknowledgement}

This work was supported by the National Natural Science Foundation of China under Grant 61203206, the Fundamental Research Funds for the Central Universities under Grant N140405001, the Specialized Research Fund for the Doctoral Program of Higher Education of China under Grant 20120042120038, the National Science Foundation for Distinguished Young Scholars of China under Grant 61425003, the State Key Laboratory of Synthetical Automation for Process Industries under Grant 2013ZCX09, and the Natural Science Foundation of Hebei Province under Grant F2014501137.

\section{References}

[1] O. S. Wolfbeis, "Fiber-optic chemical sensors and biosensors," Anal. Chem., vol. 80, no. 12, pp. 4269-4283, May. 8, 2008.

[2] G. Yin, Y. Wang, C. Liao et al., "Simultaneous refractive index and temperature measurement with LPFG and liquid-filled PCF," IEEE Photon. Technol. Lett., vol. 27, no. 4, pp. 375-378, Feb. 2015.

[3] A. N. Starodumov, L. A. Zenteno, D. Monzon, and E. De la Rosa, "Fiber Sagnac interferometer temperature sensor," Appl. Phys. Lett., vol. 70, no. 1, pp. 19-21, Jan. 1997.

[4] W. Jin, H. Xuan, C. Wang, W. Jin, and Y. Wang, "Robust microfiber photonic microcells for sensor and device applications," Opt. Exp., vol. 22, no. 23, pp. 28132-28141, Nov. 2014. 
[5] H. Y. Choi, K. S. Park, S. J. Park, U.-C. Paek, B. H. Lee, and E. S. Choi, "Miniature fiber-optic high temperature sensor based on a hybrid structured Fabry-Perot interferometer," Opt. Lett., vol. 33, no. 21, pp. 2455-2457, Nov. 2008.

[6] B. Sun et al., "Simultaneous measurement of pressure and temperature by employing Fabry-Perot interferometer based on pendant polymer droplet," Opt. Exp., vol. 23, no. 3, pp. 1906-1911, Feb. 2015.

[7] J. Wang, S. Wu, and W. Ren, "Simultaneous measurement of refractive index and temperature using an epoxy resin-based interferometer," Appl. Opt., vol. 53, no. 33, pp. 7825-7830, Nov. 2014.

[8] S. Zhang et al., "Temperature characteristics of silicon core optical fiber Fabry-Perot interferometer," Opt. Lett., vol. 40, no. 7, pp. 1362-1365, Apr. 2015.

[9] P. Lu and Q. Chen, “Asymmetrical fiber Mach-Zehnder interferometer for simultaneous measurement of axial strain and temperature," IEEE Photon. J., vol. 2, no. 6, pp. 942-953, Dec. 2010.

[10] D. Wu, T. Zhu, K. S. Chiang, and M. Deng, “All single-mode fiber Mach-Zehnder interferometer based on two peanut-shape structures,” J. Lightw. Technol., vol. 30, no. 5, pp. 805-810, Mar. 1, 2012.

[11] Y. Zhao, Y. Zhang, R. Lv, "Simultaneous measurement of magnetic field and temperature based on magnetic fluid-infiltrated photonic crystal cavity,” IEEE Trans. Instrum. Meas., vol. 64, no. 4, pp. 1055-1062, Apr. 2015.

[12] B. Sun, Z. Zhang, W. Wei, C. Wang, C. Liao, L. Zhang, and Y. Wang, "Unique temperature dependence of selectively liquid-crystal-filled photonic crystal fibers," IEEE Photon. Technol. Lett., vol. 28, no. 12, pp. 1282-1285, Jun. 2016.

[13] P. S. Reddy et al., "Method for enhancing and controlling temperature sensitivity of fiber Bragg grating sensor based on two bimetallic strips," IEEE Photon. J., vol. 4, no. 3, pp. 1035-1041, Jun. 2012.

[14] C. Park, K. I. Joo, S. W. Kang, and H. R. Kim, "A PDMS-coated optical fiber Bragg grating sensor for enhancing temperature sensitivity," J. Opt. Soc. Koreav., vol. 15, no. 4, pp. 329-334, Dec. 2011.

[15] X. Shu, L. Zhang, and I. Bennion, "Sensitivity characteristics of long period fiber gratings," J. Lightw. Technol., vol. 20, no. 2, pp. 255-266, Feb. 2002.

[16] S. Torres-Peiró, A. Díez, J. L. Cruz, and M. V. Andrés, "Sensor applications based on the cutoff properties of liquid-filled Ge-doped microstructured fibers," IEEE Sensors J., vol. 10, no. 7, pp. 1174-1179, Jul. 2010.

[17] Y. Xue et al., "Ultrasensitive temperature sensor based on an isopropanol-sealed optical microfiber taper," Opt. Lett., vol. 38, no. 8, pp. 1209-1211, Apr. 2013.

[18] G. Liu, M. Han, and W. Hou, "High-resolution and fast-response fiber optic temperature sensor using silicon Fabry-Pérot cavity,” Opt. Exp., vol. 23, no. 6, pp. 7237-7247, Mar. 2015.

[19] H. R. Iván, M. H. David, M. H. Carlos, M. H. David, and V. Joel, "Highly sensitive temperature sensor based on a polymer-coated microfiber interferometer," IEEE Photon. Technol. Lett., vol. 27, no. 24, pp. 2591-2594, Dec. 2015. 
[20] J. Luo et al., "A magnetic sensor based on a hybrid long-period fiber grating and a magnetic fluid," IEEE Photon. Technol. Lett., vol. 27, no. 9, pp. 998-1001, May. 2015.

[21] R. Yang et al. "PDMS-coated S-tapered fiber for highly sensitive measurements of transverse load and temperature," IEEE Sensors J., vol. 15, no. 6, pp. 3429-3435, Jun. 2015.

[22] C. Markos, K. Vlachos, and G. Kakarantzas, "Bending loss and thermo optic effect of a hybrid PDMS/silica photonic crystal fiber," Opt. Exp., vol. 18, no. 23, pp. 24344-24351, Nov. 2010. 